文章编号：1673-9493 (2017) 01-0040-07 中图分类号：F299.2 文献标识码: A DOI : 10.22217/upi.2016.498

\title{
国内外违法建设治理法律制度借鉴
}

\section{A Reference of the Law System of Illegal Construction Management at Home and Abroad}

摘要: 针对我国实施违法建设有效治理存在的 治理理念不清晰、法律制度不健全、行政程序 不规范、执法行为不严格等问题, 本文有针对 性地梳理了国内外有关违法建设的治理思想与 法律特征、治理主体与执行程序、具体措施与 处罚规定等方面的内容, 破解相关法律难题, 为我国违法建设治理法治化、制度化、规范化 提供经验借鉴。

Abstract: In view of the problems such as unclearness of governing concept, immature of the legal system, imperfect procedures and slack law enforcement in the process of governing illegal constructions in China, this paper combs the governance idea and legal characteristics, governing subjects and procedures, specific measures and penalty provision at home and abroad. By cracking the relevant legal problems, the paper hopes to provide some useful references for the management of illegal construction in China under the rule of law and institutionalization.

关键词: 违法建设; 法律制度; 治理手段

Keywords: Illegal Construction; Law System; Management Methods

作者: 刽懈丽, 博士, 中国人民大学公共管理学 院副教授, 教授级高级规划师。 guiyanli950525@163.com 李伟, 中国人民大学公共管理学院城乡发 展与规划专业, 硕士研究生。 383212786@qq.com
我国内地的行政执法和司法实践中，违法建设是受多个法规约束的竞合性违 法行为 ${ }^{[1]}$ 。违法建设并没有统一的司法界定和解释, 不同的法规对违法建设行为 做出了不同的规定。违法建设治理主要依靠行政手段, 各行政主管部门和地方政 府按照各自的职责分工, 分别行使违法建设相应的查处权。基于权利更迭、力量 变换以及发展导向转变等原因, 违法建设治理呈现时紧时松的局面。由于违法建 设的治理理念不清晰、法律制度不健全、行政程序不规范、执法行为不严格, 法 律的威慑力受到挑战。随着社会复杂性和不确定性的增加, 社会管理主要通过权 力和法律两种力量实现 ${ }^{[2]}$ 。法律之所以重要, 在于管理型政府不仅依靠权力, 而 且依靠法律实现对整个社会的管制。本文通过对国内外违法建设治理法律制度的 总结, 结合内地违法建设治理过程中的法律瓶颈进行梳理, 以期借鉴。

\section{1 违法建设治理思想与法律特征}

发达国家和地区对城市规划一直非常重视，根据本国和地区的情况制定了不 同法律规范、规章制度和政策办法, 采取较多措施保证城市发展在良好的规划设 计框架内运行, 尤其是对违法建设项目采取严格的治理措施, 虽然形式多样, 但 往往具有相同或类似的特征。

\section{1 违法建设的界定清晰规范}

\subsection{1 立法清晰}

与内地违法建设概念的行政界定完全不同, 发达国家和地区往往具有清晰、 明确的法律界定, 一般可分为两种类型。一是相关治理法律体现在单行法中, 如 新加坡的《建筑控制法》以及中国澳门的《澳门都市建筑总章程》属于单行法, 具体规定违法建设概念和类型。二是形成相互支撑的法律体系, 如中国香港为了 规制违法建设, 形成了以“城市规划条例”、“建筑物条例”为主, “市区重建条例”、 “土地征用条例” 等条例为辅的一系列完备的法律体系; 中国台湾根据其 “建筑 法”专门制定了“违章建筑处理办法”。

\subsection{2 规制范围明确}

发达国家和地区在专门或相关法律中对违法建筑的概念、违法建设的情形以 及 “违例” 和 “犯罪” 的标准做出了清晰界定。香港屋宇署规定 “凡未经屋宇署 
事先批准或没有通过（小型工程监管制度）的简化程序进行 的楼宇加建或改动, 均属违建工程” ${ }^{[3]}$ 。明确规制范围使得 违法建筑和违法建设的界定更加准确, 减少了法律中的模糊 地带, 也使得治理主体的工作内容更有针对性。

\section{2 强调专业人员与公众参与}

\subsection{1 强调专业人员的责任}

随着社会分工的加快, 知识结构的单一化、条块化使各 领域间形成严重的专业壁垒, 因此, 发达国家和地区越来越 强调专业人员的知识支撑。如中国香港和中国台湾均奉行 “专 业人士提供专业服务”。中国香港法律规定, 在新建或改建建 筑的时候, 业主必须委聘相关专业人士, 必要时还应按照《建 筑物条例》的相关规定聘请注册结构工程师拟备图则, 提交 屋宇署审核批准。如果建筑工程涉及岩土, 还须由注册岩土 工程师拟备图则, 提交建筑事务监督审核批准, 并且必须由 注册的承建商承建。注册结构工程师、注册岩土工程师、注 册承建商等专业注册人士都有法律规定的责任监督该建筑工 程, 如违例则会遭到检控或受纪律处分 ${ }^{[4] 140}$ 。而中国台湾 “违 章建筑处理办法” 同样规定, 对参与违法建设的建筑师、营 造业及土木包工业设计、监造或承建商, 应依法进行处罚 ${ }^{[5]}$ 。

\subsection{2 重视公众参与的作用}

发达国家和地区对于违法建设的整顿治理不仅需要专 业机构和人员的参与，而且非常强调公众的监督举报对发 现、纠改违法建设起到的重要作用。通过对公众参与的鼓 励和引导, 降低违法建筑处理的经济和社会成本, 提高行 政效率。如新加坡建设局将执业工程师例行安全检查以及 公众举报作为立案查处违法建设的信息来源 ${ }^{[6] 123}$; 中国香港 政府则强调建立共同责任制度，鼓励普通市民举报违法建 设。屋宇署设立热线 “1823 电话中心”, 每天 24 小时开通, 由该中心统筹处理每月接获的数以千计的举报 ${ }^{[4] 139}$ 。

\section{3 多维度考量违法成本收益}

违法建设多在利益驱动下发生, 即建设主体希望在有限 的空间范围内取得更大的使用权, 为此不惜忽略安全、健康 与舒适度。在违法建筑已经存在的情况下, 如何处理这些违法 建筑是一个比较重要的问题。发达国家和地区违法建设治理的 法律制度中体现的一个重要思想就是违法成本大于收益, 治 理机构往往会从经济和社会两个角度考虑违法成本与收益。

\subsection{1 经济考量}

如果违法建筑并未严重侵犯公共安全与他人权利, 只是 出现程序上的瑕疪, 为减少社会成本的浪费, 建筑方可将违
法建筑合法化, 但必须补足手续, 缴纳沉重赋税, 抵消因违 法或违章带来的收益。如德国实行了实质性违法搭建分类处 理办法: 如果违法建筑属于法律规定的尚可存在范围, 只是 业主建造前未办理审批手续, 政府往往要求业主补办手续, 颁发建设许可, 然后对业主违法建设的行为课以一定数额的 行政罚款。中国澳门的《澳门都市建筑总章程》第 70 条对 违法建设合法化的税收做出了规定：“对于无准照进行的工 程, 或因扩大建筑面积而与经核准计划不符合所进行的工程, 为使工程合法化, 所征税项为平常税款之 3 倍” ${ }^{[6] 135}$ 。反之, 如果经济上的违法成本小于收益, 建设方会将惩戒性的罚款 或赋税异化为 “花钱买许可”, 反而会造成变相鼓励的后果。

\subsection{2 社会考量}

除了经济上的考量, 发达国家和地区更加重视保护社会 利益和公众权利。以德国为例, 如果违法建筑不符合法律规定 的要求, 属于不允许存在范围, 即违法性质严重的, 地方政府 行政执法部门会责令业主自行拆除该违法建筑; 若业主不拆除, 行政执法部门组织队伍予以强制拆除。第二种情况多发生在违 法建筑严重侵犯公共安全与公共利益时。又如中国澳门的《澳 门民法典》第 1267 条规定：“任何工作物、设施, 或腐蚀性或 危险性物质之储存, 导致相邻之房地产有受不法损害之虞者, 不动产所有人即不得在其房地产内建造或保留该等工作物、设 施或储存。如该等工作物、设施或储存已获有权限之公共实体 许可, 或已符合法律为其建造或保留所规定之特别条件, 则仅 于损害已实际发生时起, 方可将其拆除和迁移。在上述任一情 况下, 就他人所遭受损失均须作出赔偿。” [7]

\section{2 违法建设治理主体与执行程序}

\section{1 监管主体广泛}

发达国家和地区违法建筑的监管主体可划分为政府机关 联动、公务人员稽查、专业人员监管、业主自律和公众监督 等五种类型。监管并非单一主体作用, 而是多元主体相互配 合的过程。如新加坡建设局将执业工程师例行安全检查以及 公众举报作为立案查处违法建设的信息来源。同时由建设局、 消防局、市区重建局对违法建设的发现、检测和审报审查等 进行联合监管, 消防局具体负责消防安全行政管理, 市区重 建局主要负责规划许可。新加坡住屋发展局根据需要, 在新 加坡建设局的指导下, 对组屋区的违法建设进行日常检查和 监管整治, 属于监管违法建设的重要参与部门 ${ }^{[6] 123}$ 。

\section{2 治理主体多元}

发达国家和地区违法建筑的治理主体主要包括行政机关 治理和社会共治两种类型。行政机关治理又可以分为专门行 
政机关治理和一般行政机关治理。新加坡和中国澳门主要通 过专门行政机关对违法建设进行查处治理, 其专门的治理机 构分别是新加坡的建设局、中国澳门的土地工务运输局。有 的国家则通过具有查处治理权限的一般行政部门进行治理, 如日本和韩国的行政厅、德国的行政官署等。

相比较而言, 中国香港更加注重多元力量的参与, 强调 社会共治, 通过建立共同责任制度, 将市民、业主、专业人 士和政府部门皆纳人遏止违法建设的体系, 形成治理合力 : 一是市民监督。政府鼓励普通市民举报违法建设; 二是业主 自律。业主有责任确保楼宇的公用和私人地方没有违法建设, 不致影响楼宇安全; 三是专业人士提供专业服务。新建或改 建建筑时业主委聘的相关专业人士, 如注册结构工程师、注 册岩土工程师、注册承建商等专业注册人士都有法律规定的 责任监督该建筑工程, 如违例就会遭到检控或受纪律处分; 四是建立部门间协调联动机制。香港政府建立违法建设治理 的联动协调机制, 违法建设行为一旦被发现, 各相关的部门 会在牵头部门 (屋宇署) 将违法者的信息发布之后, 采取行 政执法, 法院和警署则采取联合行动, 保证违法建设治理工 作落到实处; 五是屋宇署公开评估, 接受公众监督。屋宇署 每年年初都会对市民做出服务承诺, 让市民对照监督, 年终 进行自评, 以不断改进自己的服务。同时审计署对屋宇署的 工作也进行不定期的公开评估 ${ }^{[4] 140}$ 。

\section{3 执行程序完备}

发达国家和地区往往在对违法建设进行治理的整个实施 过程中对治理过程做出清晰、明确的规范。治理过程大概包括 信息收集一检测审查一性质判定一实施治理举措一法律救济 等环节, 每个环节可能涉及不同的机构和部门。尤其在实施治 理举措方面有明确的法律规定, 授予规划行政主管部门应有 的行政强制权, 并以规划部门责令停止建设的时间作为分界, 之前行为只视为违法, 不视为犯罪, 之后的建设行为即为犯 罪。如英国 1990 年《城市规划法》 (Town and Country Planning Act）以立法形式授权地方规划局行使发布实施 “强制执行通 告” 的权力。根据这一处罚手段, 一旦强制执行是事实, 非 法开发的项目必须停止建设并拆除已完成的工程。如开发者 拒不执行强制执行通告, 则违法开发构成刑事犯罪 ${ }^{[8]}$ 。

\section{4 法律救济完善}

除了强调对违法建设的处罚, 发达国家和地区还强调 对相关处罚行为的救济。如新加坡《建筑控制法》第 19 条 规定：“当事人对有关部门作出处理的具体行政行为不服的, 可向法院提起诉讼” [9]。中国台湾 “行政执行法” 则强调 “代 履行费用逾期未缴纳的, 根据法律规定移送行政执行处依本
法第二章的规定执行。也就是按照 “行政执行法” 第二章公 法上金钱给付义务之执行的规定执行。义务人对是否负担代 履行费用或者对代履行金有异议的, 可依法提起诉愿或者行 政诉讼来救济” ${ }^{[10]}$ 。另外, 中国台湾 “行政执行法” 中一个 很大的不同就在于它规定了执行时效制度。其中第 7 条规定 “行政执行, 自处分、裁定确定之日或其他依法令负有义务 经通知限期履行之文书所定期间届满之日起, 五年内未经执 行者, 不再执行; 其于五年期间届满前已开始执行者, 仍得 继续执行。但自五年期间届满之日起已逾五年尚未执行终结 者, 不得再执行。前项规定, 法律有特别规定者, 不适用之”[10]。 此条规定也适用于代履行制度。

英国的规划许可制度中规定，对于没有取得规划许可的 开发, 英国的地方政府可以视情形要求开发者补办许可申请 或采取执法行动。如果地方当局认为这种开发破坏了规划则 发出 “执法通知书”, 明确规定停工、全部拆除或部分拆除 所涉及的建筑物。如果开发者没有按规划许可的条件进行建 设, 政府则会发出“违规建设纠正通知书”, 以要求开发者 遵守规划许可的条件。开发者可以对 “执法通知书” 提出上 诉, 但不能对 “违规建设纠正通知书” 提出上诉。如果上诉 被驳回后开发者仍不遵从政府的执法决定, 则是触犯法律的 行为, 开发者因此会被政府起诉。

\section{3 违法建设治理具体措施与处罚规定}

发达国家和地区对违法建设治理的具体措施与处罚规定 各有不同, 但是基本上包含前期治理、同期治理和后期治理 三个阶段。

\section{1 前期治理}

前期治理主要是指在建筑建造前, 建造主体应向主管部 门就相关建设计划提出申请审核, 事前协调好本建筑与他建 筑、个人权利与公共权利之间的关系, 减少拆除、罚款发生 的可能性, 以期降低社会成本的过程。前期治理主要包含预 防教育、宣传引导、规划许可等。如中国香港屋宇署的网站 上, 列明业主的责任和违法建设的危害。新加坡建设局对违 法建设的类型做出简明的图例供国民参考, 并在其官方网站 上作详细的情况说明和介绍, 以警醒民众。美国制定了一整 套完整的管理制度和无微不至的服务系统, 实施严格的建筑 许可证制度 ${ }^{[11]}$ 。并且有住宅监理委员会的跟踪、检查、监督, 无证所建的附属建筑即成违法建筑, 会被强拆 ${ }^{[12]}$ 。

英国的规划许可制度在官方的规划指南中有详细介绍, 《一般开发规则》(General Development Order) 界定了不需 要申请规划许可的小型开发活动, 并规定了相应的规划要求。 如果开发项目符合一般开发规则的要求, 开发方即可获得具 
有法律效力的开发证书, 而无需再申请规划许可。英国的规 划许可制度对 “居家营业” 作了详细的规定和限制, 一旦住 宅兼作营业场所就需要申请规划许可。同时对建筑物用途的 变动也有具体的规定, 详细说明哪些用途之间的改变需要申 请规划许可, 哪些用途之间的改变不需要申请规划许可, 而 公众的意见是规划申请能否获得批准的重要因素。在以土地 公有制为主的新加坡和中国香港, 法律严格规定楼宇业主不 得侵占公共空间，在公共空间私自搭建和改造将被处以重罚。 同时新加坡的组屋政策和中国香港的廉租房政策成熟完善, 民众刚性需求基本得到满足。

\section{2 同期治理}

同期治理是指在建筑建造过程出现不合法或不合规的现 象, 监管机构和人员及时发现并采取一定措施停止对公共安 全和他人权利的侵害的过程。同期治理的措施主要是勒令停 工或停止施工。

在中国澳门，当土地工务运输局稽查人员发现违法建 设行为时, 将着令工程所有人或其代表人暂停工程 48 小时, 倘该等人士不在场时，则命令有关负责人或负责工程的技 术员执行 ${ }^{[6] 133}$ 。中国台湾因查报、检举或其他情形发现违法 建设正在施工中, 主管建筑机关应立即勒令停工, 以减少 损失等待处理 ${ }^{[4] 141}$ 。中国香港同样在法律中对在建违法建筑 有所规定, 即 : 建筑工程在建筑事务监督的命令下须停止。 如中国香港《建筑物条例》第 23 条（1996 年第 54 号第 19 条修订）规定：“任何建筑工程或街道工程正在于违反本条 例任何条文的情况下进行, 或与曾如此进行的任何建筑工程 相关; 或正在进行的任何建筑工程可能产生危险, 或严重 偏离技术备忘录、建工计划书, 或批准时附加条件不能获 得遵从, 则建筑事务监督可藉向注册一般建筑承建商、注 册专门承建商、注册小型工程承建商或进行建筑工程或街 道工程的其他人送达的书面命令, 规定停止工程。如建筑 事务监督命令停止工程, 则进行建筑工程或街道工程的人 须尽快和在尽量安全的情况下停止进行工程（由 1996 年第 54 号第 19 条增补)" ${ }^{[13]}$ 。

\section{3 后期治理}

后期治理是在违法建设中止或终止后, 治理主体在衡量 危害性程度后对违法主体和违法建筑做出具体处罚措施的 过程。

\subsection{1 对违法主体的治理措施}

违法主体可分为建设主体和专业人员。发达国家和地区 对违法主体的处罚手段包括连带责任、罚款和监禁。
连带责任制度实施的典型地区为中国香港和中国台湾。 如香港法律规定新建楼宇或加建和改建聘请的注册结构工程 师、注册岩土工程师、注册承建商都有责任监督该工程, 违 法会遭受检控或受纪律处分 ${ }^{[4] 140}$ 。台湾 “违章建筑处理办法” 规定, 对参与违法建设的建筑师、营造业及土木包工业设计、 监造或承建商, 应依法进行处罚。

罚款与监禁制度实施的典型国家和地区为新加坡、中国 香港和中国澳门。新加坡《建筑控制法》第 19 条规定：“任 何受到拆毁、移除或改变建筑物命令的人若不遵守此命令, 应受到 2 万新加坡元以下罚金或 6 月以下监禁或并罚 ; 在违 反规定的情况下, 每多一天罚款 500 新加坡元以下, 而且在 判决后如果继续违反规定, 此专业人员就会受到每天罚金不 超过 1000 新加坡元的处罚”。以上规定适用于任何建筑工程, 不管与临时建筑物有没有关系。第 20 条规定：“任何人开始 或实施或允许或授权实施未被建筑控制处处长批准的建筑工 程规划或未被许可的建筑工程都应受到处罚, 判处 20 万新 加坡元以下罚金或 2 年以下监禁或并罚, 在违反规定的情 况下, 每多一天罚 1000 新加坡元以下, 而且在判决后如果 继续违反规定, 就会受到每天罚金 2000 新加坡元以下的处 罚”。中国香港清晰界定了 “违例”、“犯罪” 的标准, 明确 高额惩罚、监禁的手段。《香港法》第 123 章 “建筑物条例” 规定, 无论任何建筑工程如未获得香港屋宇署的批准都属于 “违例”; 而违反发展审批地区图及分区计划大纲图的规定、 违反建筑管制、违反规划事务监督和建筑事务监督的要求建 设以及占用的, 都是犯罪行为 ${ }^{[14]}$ 。香港新加或经修订的 “建 筑物条例” 所订明罪行的罚则具体为以下三类 : (1) 第 40

(1AA) 条, 任何人如未获建筑实务监督的批准及同意而进 行櫭建工程, 经定罪后最高罚则为罚款 40000 港币及监禁 2 年; 及每天罚款 20000 港币；（2）第 40 (1BA）条, 任 何人如无合理辩解而没有遵从送达给他的法定清拆令, 经定 罪后最高罚则为罚款 200000 港币及监禁 1 年; 及每天罚款 20000 港币；（3）第 40（4B）条, 任何人如无合理辩解而 阻碍业主立案法团 (法团) 为遵从由屋宇署送达给法团的命 令而展开的工程, 经定罪后最高罚则为罚款 10000 港币及 监禁 6 个月 ${ }^{[15]}$ 。

\subsection{2 对于违法建筑的治理措施}

发达国家和地区对违法建筑的处罚手段主要为赎买（或 合法化)、返回原装、拆毁移除、没收接管、代履行等。

实施 “赎买（或合法化）”手段的典型国家和地区为新 加坡、中国台湾、中国澳门等。新加坡规定, 先开工后申请 并经过审核的, 可对允许的几种情形下的建筑予以赎买 ${ }^{[16]}$ 。 同样, 中国台湾也有类似规定, 即自接到违章建筑查报人员 
报告之日起 5 日内, “直辖市”、县（市）主管建筑机关应派 员到现场实施勘查, 对认定为无须拆除, 而属于程序违法建 设的, 应当通知违法建设人于收到通知之后 30 日内, 依法 补领相关执照。中国澳门《都市建筑总章程》第 70 条对外 发建筑合法化的税收做出明确规定：“对于无准照进行的工 程, 或因扩大面积而与经核准计划不符合所进行的工程, 为 使工程合法化, 所征税项为平常税款之 3 倍” ${ }^{[6] 135}$ 。

“返回原装”类似于我国的恢复原状。如新加坡规定专 业结构工程师每 5 年对各类建筑进行结构检测, 一旦发现问 题, 将作及时整改。住户及所有人未经许可, 不得对房屋结 构进行改动, 确需改动的, 必须向建设局提出书面申请并 获得建设局的批准通知书方可实施。如果未经批准即改动, 建设局将按有关法令进行处理, 严重的将被责令 “返回原 装”[6]124。

“拆毁移除” 的应用极为广泛。如新加坡《建筑控制法》 第 19 条对 “未经授权的建筑物的拆毁” 做出规定：“任何已 经落成的建筑物或已经或正在被实施的建筑规划, 若建筑控 制处处长认为其触犯了本法规定, 就可以书面命令暂停建筑 物规划的实施、拆毁该建筑物、建筑物的规划或一些改变都 应该停止触犯本法规定; 依照前款规定发布的命令要明确指 出以下事项: 建筑物被拆毁或改变的方式, 改变开始的时限, 改变完成的时限, 改变要符合建筑控制处处长的要求” ${ }^{[6] 124}$ 。

“没收接管” 是对违法建设未实现上述处罚的接续性处 罚。如中国香港“城市规划条例”第 23 条规定如果监管人 员认为该建设项目有或曾有违例情形, 监管人员可向该建设 项目的相关负责人送达 “通知书”, 并在 “通知书” 内指明 违例情形。如果相关负责人没有按照 “通知书” 的规定恢复 原状，监管人员可以介人该项建设工程并按照 “通知书” 规 定对该项目的相关财产进行接管、移走、扣留和处置。

“代履行” 又名 “代执行”, 在德国、新加坡、韩国、日 本、中国台湾、中国香港等国家和地区惯常使用, 即一项应 由义务人做出的行为义务未被履行时, 执行机关可委托他人 完成该行为, 有关费用由义务人承担。如德国 1953 年的《联 邦行政执行法》第 10 条规定了代履行制度, 第 12 条规定了 若不能实施代履行或滞纳金的措施, 主管机关可以直接强制
的方式加以完成。新加坡在《建筑控制法》第 19 条规定了 代执行措施。日本、韩国的强制执行由代执行、强制征收、 执行罚（包括秩序罚和行政刑罚）、直接强制组成, 并专设 《行政代执行法》, 确立了代执行在整个行政强制执行制度 中的核心地位。如韩国《行政代执行法》第 2 条规定 : “作 为依据法律直接命令的行为获行政厅依据法律下达命令的行 为, 当义务者不履行可由他人替代行使的行为时, 以其他手 段难以确保其履行, 或者当放任其不履行时, 认定会对公益 带来严重的危害时, 该行政厅自行行使义务者的行为, 或是 让第三人来履行, 并向义务人征收费用”。

\section{4 违法建设治理他山之石与法律之困}

\section{1 他山之石}

新加坡、中国香港、中国台湾、德国、美国等国家和地 区在违法建设治理上取得了很大的成绩，机制健全、手段灵 活、衔接得当的违法建设责任追究制度, 多管齐下, 这与其 强有力的违法建设治理措施（表 1 ）密不可分。一是刑罚的 必定性与及时性。即违法建设治理遵循意大利刑法学家贝卡 里亚 (Cesane Bonesana Beccarra) 在其经典名著《论犯罪与 刑罚》中所言： “对犯罪最强有力的约束力量不是刑罚的严 酷性, 而是刑罚的必定性”。确定违法者不仅没有违法收益, 而且必须付出比违法收益更高的违法成本, 同时也要及时查 处 ${ }^{[17]}$ 。二是追究连带责任。即追究建筑设计师及承建商等相 关人员的连带责任, 对参与违法建设的建筑设计师和承建商 等人员依法进行处罚。三是提高违法成本。发达国家和地区 或通过高额罚金, 或通过征收高额税金来提高违法建设的成 本, 从而达到抑制违法建设的目的。四是违法建设人刑。违 法建设人刑是针对违法建设行为相对人的处罚, 其威慑作用 最大, 并实现行政处罚与刑事处罚的有效衔接。五是鼓励公 众参与。发达国家和地区违法建设治理中最显著的特点是奖 惩分明, 执法严厉, 法律宣传和社会广泛监督相协调, 具有 市民互动参与的路径和方法, 公众成为利益相关者。六是实 施代履行制度。治理违法建设行政代执行对象的义务都是由 法律直接规定或者由执法主体依据法律下达命令, 且他人可 以代为执行, 从而促进执法成本降低和执法效率提高。七是

表 1 违法建设治理手段比较

\begin{tabular}{|c|c|c|c|c|}
\hline & 中国内地 & 新加坡 & 中国香港 & 中国澳门 \\
\hline 罚款 & $\begin{array}{l}\text { 工程造价 } 10 \% \text { 以下, 个人不超过 } \\
10 \text { 万元, 单位不超过 } 100 \text { 万元 }\end{array}$ & $\begin{array}{l}\text { 不分单位个人, 可处 } 20 \text { 万新加坡元以下罚款, } \\
\text { 违者每天 } 1000 \text { 2 } 000 \text { 新加坡元滞纳金 }\end{array}$ & $\begin{array}{l}\text { 首次 } 50 \text { 万港币, 以后 } 100 \text { 万港币, } \\
\text { 持续犯每日不超过 } 5000 \text { 港币 }\end{array}$ & $1000 \sim 20000$ 澳门元 \\
\hline 合法化 & 限期改正；依据时间早晚确权 & 赎买 & - & 征收平常税款之 3 倍 \\
\hline 刑事处罚 & - & 罚款和监禁, 可并处 & 罚款和监禁, 可并处 & - \\
\hline 承建商责任追究 & - & - & 追究承建商责任 & - \\
\hline
\end{tabular}


注重法制宣传。在发达国家和地区, 既有建筑物进行改建或 扩建时需经批准是作为常识存在于普通民众心中的, 这与法 制基础和宣传教育直接相关。

\section{2 法律之困}

实践过程中, 我国内地实施违法建设的有效治理尚存在 一些法理认识困境和缺乏制度配套问题, 需要借鉴发达国家 和地区的法律制度并加以完善。

一是违法建设的法律界定问题。违法建设是行政管理 中的常用概念, 在国家立法层面并无 “违法建设” 的专属概 念和相关定义。行业法中 “违法建设” 概念对应违反规划行 政许可规定的行为, 是违反规划控制导致空间结构改变的建 筑物和构筑物修建活动。理论层面, 城市规划作为一种追求 未来理想和效用的社会实践, 其目标的多样性和超前性无法 使社会成员确信将来从中受益, 因此社会整体对规划的自愿 服从成为一种不可能。因此违法建设是一种无法通过 “行为自 觉” 而得到遏制的现象, 必须由国家强制力予以规范和约束 ${ }^{[18]}$ 。 为有效遏制违法建设的蔓延, 需要制定专门处理违法建设的 法律, 从法理学等角度对违法建设的要素进行分析, 根据违 法建设的性质进行合理的分类和法律评价, 对违法建设做科 学合理的认定和处理, 由不履行法律义务的行为相对人承担 相应的法律责任 ${ }^{[19]}$ 。

二是违法建设人刑的合理解释问题。违法建设侵害对远 期的社会环境效益受损和区域发展机会丧失等非显性伤害, 与对人或物的直接现实侵害相比并不显见。由于在违法建设 治理过程中会出现竞合犯罪问题，与违法建设相关的犯罪类 型有非法占用农用地罪、非法转让倒卖土地使用权罪、重大 环境污染事故罪、妨害公务罪、煽动暴力抗拒法律实施罪、 聚众冲击国家机关罪、非法经营罪以及渎职罪 ${ }^{1}$ 等八种。同 时, 按照《侵权法》规定的民事权益, 与违法建设相关的有 生命权和健康权, 即可能由于违法建设遮挡阳光、破坏消防 通道等而产生的生命权和健康权侵害, 遗憾的是该法并未对 违法建设侵害进行详细规定。也就是说，现有的刑罚制度对 违法建设侵害公共利益的危害和损失缺乏法理的有效解释。 我国违法建设历史存量巨大, 其逐步消解需要在现行法律制 度框架内运用政治智慧和经济手段, 采取在建违法建设人刑 是从根本上遏制新的违法建设的可行手段 ${ }^{[20]}$ 。因此应借鉴发 达国家和地区的司法经验进行刑事处罚规定, 在《刑法》中 增设违法建设罪及相应条款, 对不接受行政处罚仍继续违法 建设的违法主体课以刑罚, 对施工、设计方追究连带责任。
三是新生违法建设的司法解释问题。《行政强制法》第 44 条规定强制拆除并未涉及正在进行违法建设。因此针对 新生违法建设的强制拆除存在行政强制措施和行政强制执行 两种观点, 而是否存在行政决定作为区分标准。由于《行政 处罚法》中规定, 没有法定依据或者不遵守法定程序的行政 处罚无效, 因此城市管理及规划建设行政主管部门面对众多 不自觉履行行政处罚的违法建设束手无策, 缺乏强制力, 行 政效率受到侵害 ${ }^{[10]}$ 。新生违法建设属于正在发生的违法行为, 其行为正在一步步危害社会公共利益, 因此, 将新生违法建 设及时拆除, 是对社会公共利益的正当防卫, 应对此执行特 别行政执行程序。

四是代执行制度实施的配套制度问题。德国代履行制度 规定, 代履行费用在事先的告诫中应当列明预定的数额。实 际的费用超过告诫所告知的数额时, 不影响有关债务的追索 权。日本的《行政代执行法》规定了义务人在拒绝缴纳费用 时, 行政厅能够依照国税滞纳处分进行强制征收。我国《行 政强制法》并没有规定义务人若是拒绝缴纳费用行政机关能 够采取什么措施来征收费用。一般而言, 如果当事人拒绝支 付代履行费用, 那么第三方可向行政机关行使支付报酬的请 求权, 行政机关在支付第三方相关费用后享有对义务人的返 还请求权 (公法请求权)。行政机关的公法请求权可通过强 制执行实现, 具体方式为划拨当事人存款、汇款或者通过拍 卖当事人财物抵扣费用。但行政机关划拨当事人存款、汇款 需要银行等金融机构的配合, 而在实际操作中, 银行等金融 机构考虑到自身利益, 会拒绝行政机关划拨当事人存款、汇款, 这就造成代履行的费用难以受偿。同时由于违法建设为不可 移动财产, 在没有合法化之前不能拍卖。对于不符合规划要 求的违法建设, 之所以要没收是因为 “无法拆除”。但现有法 律缺乏对于无法拆除而又不符合规划的违法建设的具体处理 规定, 即如何通过一定程序使之合法化并保障待执行费用的 征收, 同时作为公益使用或公开拍卖都需要规范化制度。

五是处罚竞合的实际运用问题。违法建设查处过程中, 同一违法建设行为往往会触犯不同法律、法规和规定, 在 违法处罚上涉及多个行政执法部门，这就涉及违法竞合问 题, 其处罚原则是从重处罚, 即由处罚最重的部门给予处 罚。但在实际操作中, 查处部门有可能不是处罚最重的部门, 而查处部门依照自身职责给予处罚后, 根据行政法 “一事 不再罚” 原则, 处罚最重的部门不能进行二次处罚, 这就 致使违法建设逃脱了本应有的严厉惩罚。如何设置案件移 送的违法竞合前置处理程序, 从而将案件移交处罚最重的

(1) 渎职罪是针对国家工作人员的罪责, 主要有㿟私舞整不移交刑事案件罪、玩忽职守罪、滥用职权罪、非法批准征用占用土地罪、非法低价出 让国有土地使用权罪等。 
行政部门, 成为一个重大问题, 涉及程序要件的构建 : 一 是对复杂违法建设的认定采取联合认定机制；二是由裁判 部门裁定最重处罚部门进行查处；三是遵循比例原则，有 效减少拆除和没收等手段造成相对方的损失, 界定政府应 承担的责任部分 ${ }^{[21]}$ 。 UP

\section{参考文献}

[1] 孟骁, 欧伟光. “违建入刑” 是减少违法建设乱象的有效途径 [J]. 法制 与社会, 2015, 32: 190-191.

[2] 张乾友. 变革社会中的服务型政府建设一任务型组织的途径 [J]. 北 京行政学院学报, 2014, 1: 43-49.

[3] 香港特别行政区屋宇署. 我们的服务一楼宇安全一违例建筑工程 [EB/OL]. [2016-12-10]. http://www.bd.gov.hk/sc/services/index_exist2. html\#UBW1.

[4] 沈晖, 陈瑶. 境外治理违法建筑的经验和启示 [J]. 湖南大学学报（社 会科学版)，2012, 6: 138-142.

[5] 法邦网. 《违章建筑处理办法》第 10 条 [EB/OL]. (1999-06-29)[201612-16]. http://code.fabao365.com/law_55542.html.

[6] 蒋拯. 违法建筑处理制度研究一一权利保护与限制的视角 [D]. 西南 政法大学, 2012.

[7] 澳门特别行政区政府印务局. 民法典第 1267 条 [EB/OL]. (1999-0803)[2016-12-10]. http://bo.io.gov.mo/bo/i/99/31/codciven/codciv1201. asp\#13t2.

[8] 邓迪敏. 对违法建设的法律思考 [J]. 城市规划, 2000, 10: 14-16, 20.

[9] Building and Construction Authority. Codes, Acts and Regulations Building Control Act, § 19, Chapter 29[S]. (1999-12-30)[2016-12-10]. Singapore Government. https://www.bca.gov.sg/BuildingControlAct/ others/building_control_act.pdf.

[10] 中国宪政网.“台湾地区行政执行法” [EB/OL]. (1999-10-22)[2016-1210]. http://www.calaw.cn/article/default.asp?id=3177.

[11] 看国外如何治理违法建筑 [J]. 中外房地产导报, 2002, 23: 32-34.

[12] 马柏成. 借鉴美国城市管理经验, 建立城市管理联动机制 [J]. 城市监 察, 2007, 4: 50-53.

[13] 香港律政司双语法律资料系统. 建筑物条例第 23 条 [EB/OL]. [201612-10]. http://translate.legislation.gov.hk/gb/www.legislation.gov.hk/09/ chi/pdf.htm.

[14] 香港律政司双语法律资料系统. 建筑物条例第 40(1BA) 条 [EB/OL]. [2016-12-10]. http://translate.legislation.gov.hk/gb/www.legislation.gov. hk/09/chi/pdf.htm.

[15] 香港特别行政区屋宇署. 2004 年建筑物 (修订) 条例 - 注意事项及答 与问 - 订明罪行的修订罚则 [EB/OL]. [2016-12-10]. http://www.bd.gov. hk/sc/BAO_notes.html.

[16] 陈春英. 新加坡管控违法建筑的七大举措 [J]. 上海城市管理职业技术 学院学报, 2008, 2: 57-58.

[17] 切萨雷. 贝卡里亚. 论犯罪与刑罚 [M]. 黄风, 译. 北京: 中国法制出版 社, 2005: 72 .

[18]范德虎, 谢谟文. 城乡规划违法建设的法律界定及其要素分析 [J]. 规 划师, 2012, 12: 61-65.

[19] 蒋拯. 违法建筑定义问题研究 [J]. 河南省政法管理干部学院学报, 2011, Z1: 157-163.

[20] 刘光圣.违法建设治理中的刑法适用 [J]. 人民检察, 2012, 4: 19.

[21] 文超祥, 张其邦, 马武定. 论违法建设行政处罚的必定性与相当性 [J]. 城市规划, 2013, 10: 47-52. 